\title{
Clinical research on minimally invasive rotary resection in the treatment of lactational breast abscess
}

\author{
Ze-Long Fü, Lin Zhang", Rui Feng, Fang-Xin Wan \\ Department of Mammary Gland, Tianjin Central Hospital of Gynecology Obstetrics, Tianjin, China \\ Contributions: (I) Conception and design: ZL Fu; (II) Administrative support: R Feng; (III) Provision of study materials or patients: ZL Fu, Rui Feng; \\ (IV) Collection and assembly of data: ZL Fu, FX Wan; (V) Data analysis and interpretation: ZL Fu, L Zhang; (VI) Manuscript writing: All authors; \\ (VII) Final approval of manuscript: All authors. \\ \#These authors contributed equally to this work. \\ Correspondence to: Rui Feng. Department of Mammary Gland, Tianjin Central Hospital of Gynecology Obstetrics, No. 156, Nankai Third Road, \\ Nankai District, Tianjin 300100, China. Email: dr.fr@126.com; Fang-Xin Wan. Department of Mammary Gland, Tianjin Central Hospital of \\ Gynecology Obstetrics, No. 156, Nankai Third Road, Nankai District, Tianjin 300100, China. Email: 411814020@qq.com.
}

Background: This study sought to compare the efficacy of minimally invasive rotatory resection plus catheter drainage and puncture abscess aspiration in the treatment of lactational breast abscess

Methods: The data of 80 patients with lactational breast abscess at the Department of Mammary Gland, Tianjin Central Hospital of Gynecology Obstetrics, from January 2019 to January 2020 were retrospectively collected. The patients were divided into the minimally invasive group $(n=30)$ and the abscess aspiration group ( $\mathrm{n}=50)$. Clinical data, including age, parity, preoperative body temperature, postpartum time, time of abscess cavity generation, diameter and position of abscess cavity, intraoperative drainage, bleeding, recovery time, and satisfaction score, were collected and compared between the 2 groups of patients.

Results: The baseline data between the 2 groups were not significantly different. The minimally invasive group had a higher single drainage volume and single bleeding volume $(\mathrm{P}<0.05)$, and lower cumulative pain score $(\mathrm{P}<0.05)$; the cumulative bleeding volume did not differ significantly between the 2 groups $(\mathrm{P}>0.05)$. For the abscess cavities with a diameter $\leq 5 \mathrm{~cm}$, the single drainage volume, single bleeding volume, and single pain score in the minimally invasive group were significantly higher than those in the abscess aspiration group $(\mathrm{P}<0.05)$; the total drainage volume was similar between the 2 groups $(\mathrm{P}>0.05)$. For the abscess cavities with a diameter $>5 \mathrm{~cm}$, the single drainage volume and single bleeding volume in the minimally invasive group were significantly higher than those in the abscess aspiration group $(\mathrm{P}<0.05)$, while the cumulative bleeding volume, single pain score, and cumulative pain score were lower in the minimally invasive group $(\mathrm{P}<0.05)$. There was no significant difference in total drainage between the 2 groups $(\mathrm{P}>0.05)$. Conclusions: When the abscess cavity is $\leq 5 \mathrm{~cm}$ in diameter and the abscess is superficial, puncture and abscess aspiration may be more advantageous than the minimally invasive rotatory resection plus catheter drainage. When the abscess cavity is $>5 \mathrm{~cm}$ in diameter and there are multiple abscesses or deep large abscess, the efficacy of minimally invasive rotary resection plus catheter drainage may be better than the puncture and abscess aspiration.

Keywords: Lactational mastitis; breast abscess; ultrasound-guided puncture abscess aspiration; minimally invasive rotary resection

Submitted Sep 28, 2021. Accepted for publication Dec 15, 2021.

doi: $10.21037 /$ gs-21-729

View this article at: https://dx.doi.org/10.21037/gs-21-729 


\section{Introduction}

A lactational breast abscess is a relatively common complication of breast infection, which can cause serious clinical outcomes in postpartum women. The complaints of patients mainly include pain, fever, or breastfeeding interruption (1). The occurrence rate of mastitis has been estimated to be about $9-33 \%$ in lactating women (2); however, the figure varies due to differences in race or breastfeeding methods. Breastfeeding mastitis was the main cause leading to lactational breast abscess. Patients with lactational breast abscesses develop in about $4-11 \%$ of women with mastitis (3). Breastfeeding mastitis can cause women to stop breastfeeding (4). The common cause of the disease is a Staphylococcus aureus and surface Staphylococcus infection, the early manifestation of which is the local inflammation of the breast (5). If mastitis is not treated early, a breast abscess may develop at a later stage.

For patients with a formed abscess, the indications for surgical intervention have been established (6). Traditionally, the methods commonly used to treat lactational breast abscesses were repeated percutaneous needle aspirations or indwelling catheter drainage (7). The current preferred treatment is ultrasound-guided abscess puncture, but it is not always effective in treating abscesses with large abscess cavities, multiple abscess cavities, or many necrotic tissues (8). In recent years, the vacuum-assisted minimally invasive breast resection system has made positive progress in the treatment of breast abscesses (9). This system was originated from the minimally invasive biopsy system, which was used for diagnosis. Because it can resect the whole lesions of breast, the minimally invasive breast resection system has been used for treatment of breast diseases. However, only limited research has been conducted on this system (10), and its specific indications have not yet been closely explored.

To determine the most appropriate method for the treatment of lactational breast abscess, we investigated the intraoperative and postoperative data of patients who underwent minimally invasive rotary resection plus catheter drainage and puncture abscess aspiration. This clinical evidence should provide insights into the advantages and disadvantages of these 2 strategies, and thus help clinical surgeons make optimal decisions for patients. We present the following article in accordance with the STROBE reporting checklist (available at https://dx.doi.org/10.21037/ gs-21-729).

\section{Methods}

\section{Subjects}

This was a retrospective study. Patients diagnosed with lactational breast abscess at the Department of Mammary Gland, Tianjin Central Hospital of Gynecology Obstetrics, from January 2019 to January 2020 were included in this study. The data of 80 patients were collected. Of the 80 patients, 30 underwent minimally invasive rotary resection (the minimally invasive group), and 50 underwent puncture abscess aspiration (the abscess aspiration group). To be eligible for inclusion in the study, patients had to meet the following inclusion criteria: (I) be in the breastfeeding period, and have inflammation indicated by increased blood routine and C-reactive protein (CRP) levels; (II) have undergone an ultrasound examination of the breast that indicated galactostasis and breast abscess formation (other acute inflammatory lesions were excluded); (III) have no or only mild systemic reactions, such as chills or a body temperature $<39{ }^{\circ} \mathrm{C}$; (IV) have no coagulation dysfunction or other organ dysfunction; (V) have voluntarily agreed to the treatment regimen and receiving a postoperative follow-up telephone call. Patients were excluded from the study if they met any of the following exclusion criteria: (I) had breastfeeding mastitis but had not yet formed an obvious abscess cavity; (II) the skin of the abscess cavity had ruptured or was about to rupture; (III) had non-lactational mastitis; (IV) had a severe systemic reaction (e.g., a body temperature $>39^{\circ} \mathrm{C}$ ); (V) had severe dysfunction of other organs; and/ or (VI) had refused the treatment plan provided by our Department. All procedures performed in this study involving human participants were in accordance with the Declaration of Helsinki (as revised in 2013). The study was approved by ethics committee of Tianjin Central Hospital of Gynecology Obstetrics (No. 2021KY058) and informed consents were exempted by the ethics committee due to the retrospective nature of this study.

\section{Surgical procedures and postoperative treatment}

The surgeries of the patients in the 2 groups were conducted in an outpatient operating room. The selection of treatment method was based on the decision of the patients after fully explaining the benefits and risks of these two methods. The lidocaine required for local anesthesia was from the same manufacturer and of the same specification for the 2 groups. 

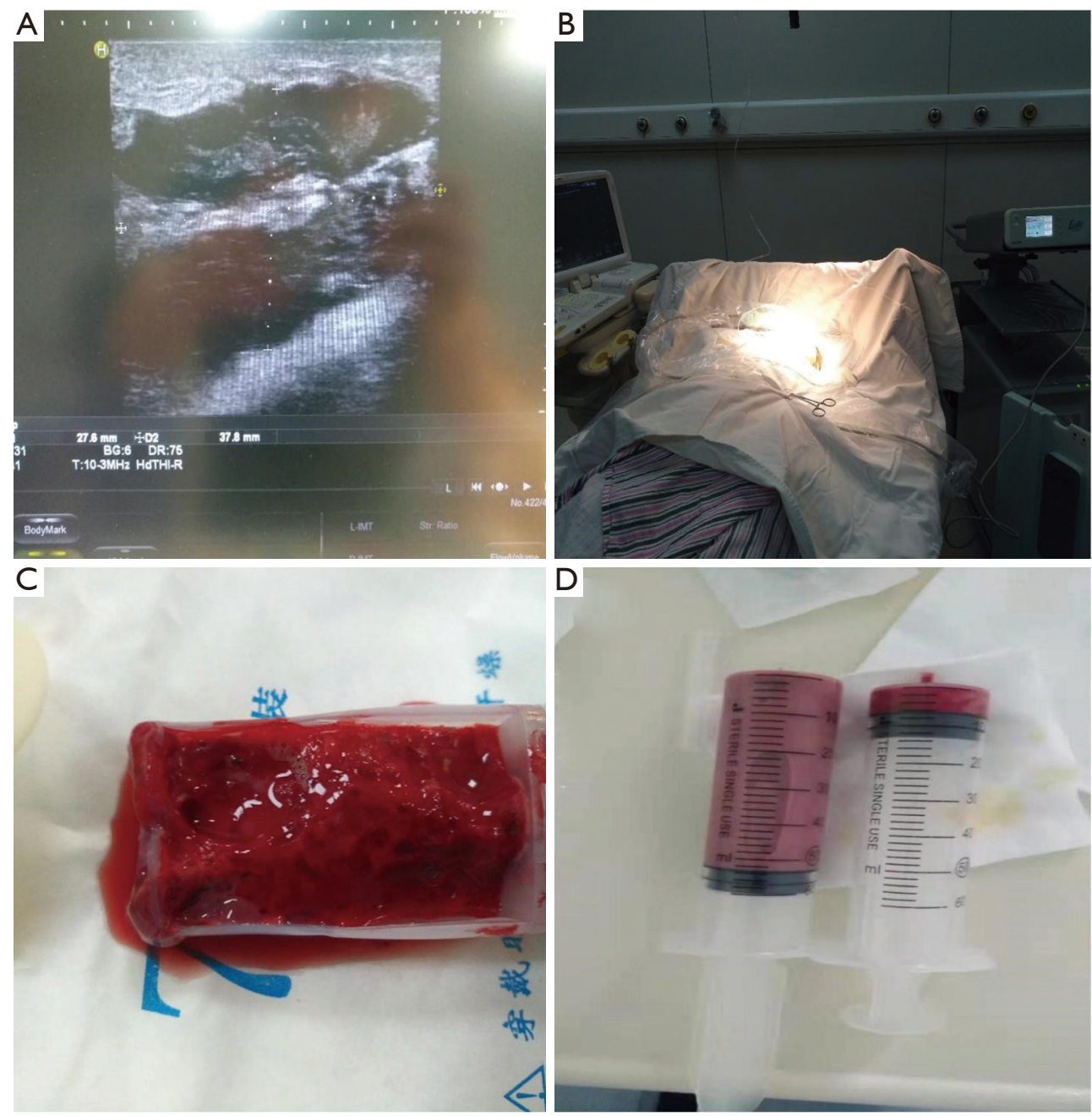

Figure 1 Representative images of surgeries and specimens. (A) Preoperative bedside color ultrasound of patient; (B) a minimally invasive rotary resection was performed; (C) specimens collected by the minimally invasive rotary cutter; (D) specimens collected by puncture abscess aspiration.

\section{Minimally invasive group}

The patient was placed in a supine position. Before surgery, the abscess cavity was located and marked by ultrasound at the bedside (Figure 1A). The incision was generally made at the normal breast near the lower edge of the abscess cavity to avoid making puncture points in the areola area. Routine disinfection and drapes were conducted. An endoscopic sleeve and a minimally invasive rotary cutter were used to ligate the bedside ultrasound probe. After local anesthesia, the infusion set was connected with $0.9 \%$ saline for use, and the steel needle of the connected infusion set was directly inserted into the upper edge of the abscess cavity. If the abscess cavity was deep, a long indwelling needle was used.
Under the guidance of the ultrasound, the rotary cutter was inserted into the abscess cavity and the vacuum suction was turned on until the entire abscess was aspirated. If there was a partition in the abscess cavity, the rotary incision mode was used to open up the separation between the abscess cavities, connecting the impassable multiple cavity separated lesions into a single cavity lesion, which was conducive to subsequent irrigation and drainage (Figure 1B). Suction was performed to remove necrotic tissues. The abscess cavity was continuously flushed with saline, and the flushing fluid was sucked until the color of the flushing fluid was clear, and there was no necrosis. The rotary cutter was removed and a drainage tube was placed at the puncture point and 
fixed with suture lines. The negative pressure drainage system was connected. The puncture point was covered with sterile gauze, and the operation was completed.

After surgery, the patient's body temperature, routine blood work, CRP level, and other indicators were recorded. If the patient's body temperature was $>38.5^{\circ} \mathrm{C}$ and white blood cell count (WBC) was $>1.2 \times 10^{9} / \mathrm{L}$ after surgery, broad-spectrum antibiotics were administered. After the bacterial culture results were received, the antibiotics were adjusted as necessary. The antibiotic medication cycle was 10-14 days. When the patient's body temperature was normal for $>3$ days and the WBC and neutrophil count returned to normal, consideration was given to stopping the medication (11). The patients were instructed to continue breastfeeding after surgery, and empty their milk every day. The patients were also asked to return to the hospital daily for dressing changes after surgery, and the properties and drainage volume of the drainage fluid in each suction ball were recorded. If the drainage volume was $<5 \mathrm{~mL} / \mathrm{d}$ (12), the drainage tube was removed. The dressing changes were continued until the incision was healed. A further breast color ultrasound examination was then conducted.

\section{Abscess aspiration group}

The patient was placed in a supine position. Before surgery, the abscess cavity was located and marked by ultrasound at the bedside (Figure 1A). The surface of the deepest abscess cavity was chosen as the puncture point, and the areola area was avoided. The ultrasound probe was ligated with the endoscopic sleeve, and conventional disinfection was performed. After administering local anesthesia, a 20 or $50 \mathrm{~mL}$ syringe was selected to puncture the abscess cavity, and the abscess in the cavity was sucked clean under ultrasound guidance. After the suction was completed, the puncture point was pressed for 510 minutes, and sterile gauze was used to cover the puncture site. The surgery was completed.

After surgery, the patient's body temperature, routine blood work, CRP level, and other indicators were recorded. If the patient met the indications for antibiotic use, a full course of antibiotics was administered. The patient was instructed to return to the hospital daily, and any changes in the abscess cavity were observed with ultrasound. If the abscess cavity was filled with abscess again, the abscess aspiration was re-performed until the abscess cavity of the patient was reduced. When the abscess cavity was $<2 \mathrm{~cm}$ (as detected by a B-ultrasound), the abscess aspiration was stopped (10). The properties and amount of abscess were recorded each time. A further breast color ultrasound examination was conducted after the abscess aspiration was stopped.

\section{Observation indicators}

The criteria for diagnosis of recovery were as follows: the disappearance of local and systemic inflammatory symptoms, no obvious liquid dark area on the ultrasound examination, and no recurrence of symptoms or signs after a 1 month follow-up period (5).

The intraoperative and postoperative indicators included the amount of drainage, the incision length, the amount of bleeding (single bleeding and cumulative bleeding), the pain score [the 0-10-point visual analogue scale (VAS), on which 0 points indicates no pain, and 10 points indicates severe pain, was used to represent different degrees of pain], the postoperative abscess culture results, the drainage time, the recovery time, the total drainage amount (intraoperative drainage plus postoperative drainage), the patient's willingness to continue breastfeeding, the continuance or discontinuance of breastfeeding, and patient satisfaction.

\section{Statistical analysis}

SPSS22.0 was used for the statistical analysis in this study. The measurement data were first tested for normality, and those with a normal distribution were expressed as mean \pm standard variation (SD). The independent $t$-test was used to compare differences between the 2 groups. The count data were represented ass the number of cases and percentage, and the $\chi^{2}$ test was used for between-group comparisons. A P value $<0.05$ was considered statistically significant.

\section{Results}

\section{Baseline data between the 2 groups of patients}

There were no significant differences in the age, parity, preoperative body temperature, postpartum time, time of abscess cavity generation, diameter of abscess cavity, and position of abscess cavity between the 2 groups $(\mathrm{P}>0.05)$. Thus, the baseline characteristics between the 2 groups were comparable (Table 1 for further details). 
Table 1 Baseline characteristics of patients between the 2 groups

\begin{tabular}{|c|c|c|c|c|}
\hline & Minimally invasive group $(n=30)$ & Abscess aspiration group $(n=50)$ & $\mathrm{t} / \chi^{2}$ & $\mathrm{P}$ \\
\hline \multicolumn{5}{|l|}{ Parity } \\
\hline Primipara & 21 & 38 & 0.350 & 0.560 \\
\hline Pluripara & 9 & 12 & & \\
\hline Postpartum time (days) & $35.63 \pm 23.10$ & $34.46 \pm 17.70$ & 0.256 & 0.799 \\
\hline Time of abscess cavity generation (days) & $10.20 \pm 4.20$ & $10.80 \pm 4.44$ & -0.597 & 0.552 \\
\hline Diameter of abscess cavity (cm) & $6.45 \pm 2.91$ & $6.40 \pm 2.27$ & 0.088 & 0.930 \\
\hline \multicolumn{5}{|l|}{ Position of abscess cavity } \\
\hline
\end{tabular}

The theoretical frequency of the abscess position was $<5$, and the results of a Fisher test were used. There were no significant differences in age, body temperature before surgery, postpartum time, abscess cavity time, and abscess size between the groups ( $P>0.05)$. There was also no statistical difference in the parity and the position of the abscess cavities between the groups $(P>0.05)$.

\section{Comparisons of intraoperative and postoperative indicators between the 2 groups}

In the minimally invasive group, the drainage fluid comprised yellow-white to off-white purulent secretions, and red necrotic tissues and viscous abscesses were observed in the postoperative specimen collection tank (Figure 1C). In the ultrasound-guided abscess aspiration group, the aspiration fluid comprised yellowish-white to grayish-white purulent secretions. It was difficult to aspirate in the later stage of the aspiration, and a small amount of blood was sometimes drawn out, such that there was a mixture of secretions and blood (Figure 1D). The minimally invasive group had a higher amount of intraoperative drainage and single bleeding, a lower cumulative pain score, and a longer incision length than the abscess aspiration group $(\mathrm{P}<0.05$; Table 2$)$, but the cumulative bleeding was similar between the 2 groups $(\mathrm{P}>0.05$; Table 2$)$. In addition, the recovery time was shorter and the satisfaction score was higher in the minimally invasive group than in the abscess aspiration group $(\mathrm{P}<0.05$; Table 2$)$.
Comparisons of intraoperative and postoperative indicators between the 2 groups for patients with an abscess cavity diameter $\leq 5 \mathrm{~cm}$

Previous research (13) has shown that the efficacy of abscess aspiration in patients with an abscesses cavity diameter $>5 \mathrm{~cm}$ was poor. Thus, we subdivided the patients into groups according to the diameter of the abscess cavity. A diameter $\leq 5 \mathrm{~cm}$ was defined as a small abscess cavity, a diameter $>5 \mathrm{~cm}$ was defined as a large abscess cavity. A further analysis was performed.

For patients with a small abscess cavity, there was no significant difference in the size of the cavity between the 2 treatment groups $(\mathrm{P}>0.05$; Table 3). The minimally invasive group had a higher amount of single drainage, a higher amount of bleeding, a higher single intraoperative pain score, and a lower postoperative cumulative pain score than the aspiration group $(\mathrm{P}<0.05$; Table 3$)$, but the cumulative postoperative pain score of the minimally invasive group was much lower than that of the puncture aspiration group. The total amount of drainage was similar between the 2 groups $(\mathrm{P}>0.05$; Table 3). Notably, patients' satisfaction 
Table 2 Comparison of surgical parameters and clinical outcomes between the 2 groups of patients

\begin{tabular}{|c|c|c|c|c|}
\hline & Minimally invasive group $(n=30)$ & Abscess aspiration group $(n=50)$ & $\mathrm{t}$ & $\mathrm{P}$ \\
\hline Intraoperative incision (cm) & $0.59 \pm 0.07$ & $0.09 \pm 0.03$ & 35.503 & $<0.001$ \\
\hline Single bleeding $(\mathrm{mL})$ & $9.5 \pm 2.74$ & $1.23 \pm 0.79$ & 16.147 & $<0.001$ \\
\hline Cumulative bleeding (mL) & $9.5 \pm 2.74$ & $10.86 \pm 6.09$ & -1.365 & 0.176 \\
\hline Recovery time (days) & $12.13 \pm 3.18$ & $15.72 \pm 6.80$ & -3.190 & 0.002 \\
\hline Satisfaction score & $7.97 \pm 0.81$ & $7.46 \pm 0.93$ & 2.473 & 0.016 \\
\hline
\end{tabular}

Table 3 Comparison of surgical parameters and clinical outcomes between the 2 groups in patients with an abscess cavity diameter $\leq 5 \mathrm{~cm}$

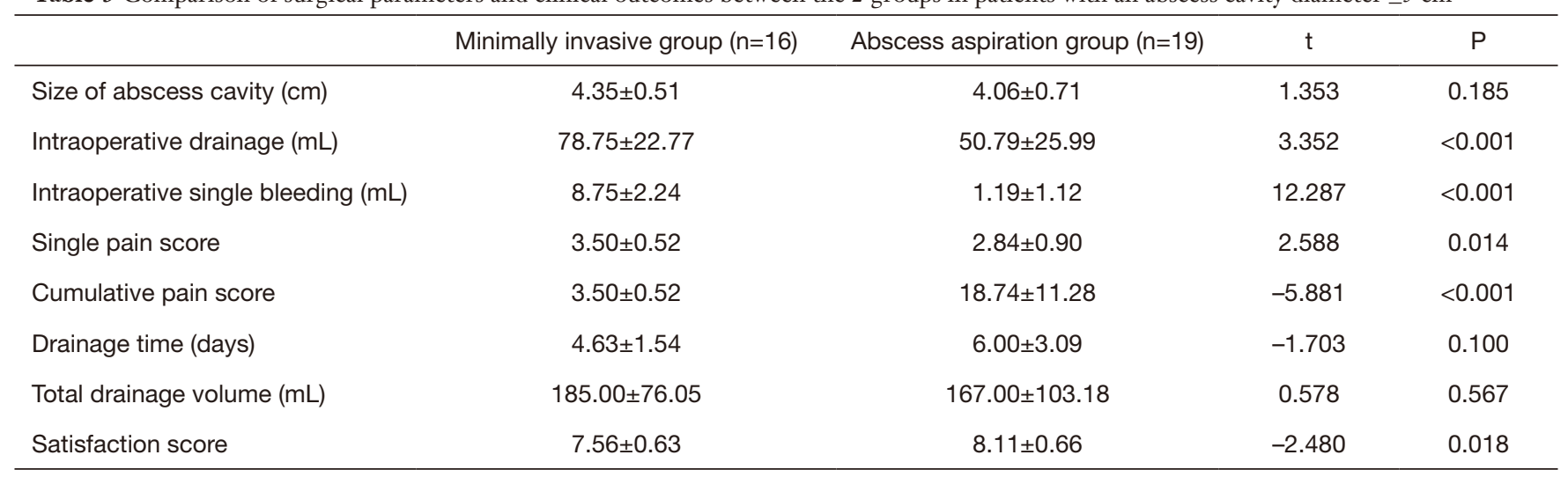

scores were lower in the minimally invasive group than the aspiration group $(\mathrm{P}<0.05$; Table 3$)$. Thus, when the diameter of the abscess cavity was $<5 \mathrm{~cm}$, the minimally invasive technique provided no significant therapeutic advantages, but was more expensive and the operation was complicated.

\section{Comparisons of intraoperative and postoperative indicators between the 2 groups for patients with an abscess cavity diameter $>5 \mathrm{~cm}$}

For patients with a large abscess cavity, there was no significant difference in the size of the cavity between the 2 groups $(\mathrm{P}>0.05$; Table 4). The amount of aspiration and single bleeding during the surgery was higher in the minimally invasive group than that in the abscess aspiration group, but the postoperative cumulative bleeding was less in the minimally invasive group than that in the abscess aspiration group. Further, the intraoperative single pain and cumulative pain scores were lower in the minimally invasive group than the abscess aspiration group
$(\mathrm{P}<0.05$; Table 4). In addition, the minimally invasive group had a shorter drainage time and recovery time, and a higher satisfaction score than the abscess aspiration group $(\mathrm{P}<0.05$; Table 4$)$. The total amount of drainage was similar between the 2 groups $(\mathrm{P}>0.05)$.

As the curve in Figure 2 shows, the postoperative drainage volume was not steady and declined rapidly, and for some patients, the post-surgery drainage volume was similar to or even more than the initial drainage volume.

\section{Discussion}

In this study, we compared the efficacy of minimally invasive rotary resection and puncture abscess aspiration in the treatment of lactational breast abscess by investigating intraoperative and postoperative indicators. The results showed that patients in the minimally invasive group had higher amounts of drainage and bleeding, and a lower cumulative pain score than those in the abscess aspiration group. A further analysis was conducted by subdividing 
Table 4 Comparison of surgical parameters and clinical outcomes between the 2 groups in patients with an abscess cavity diameter $>5 \mathrm{~cm}$

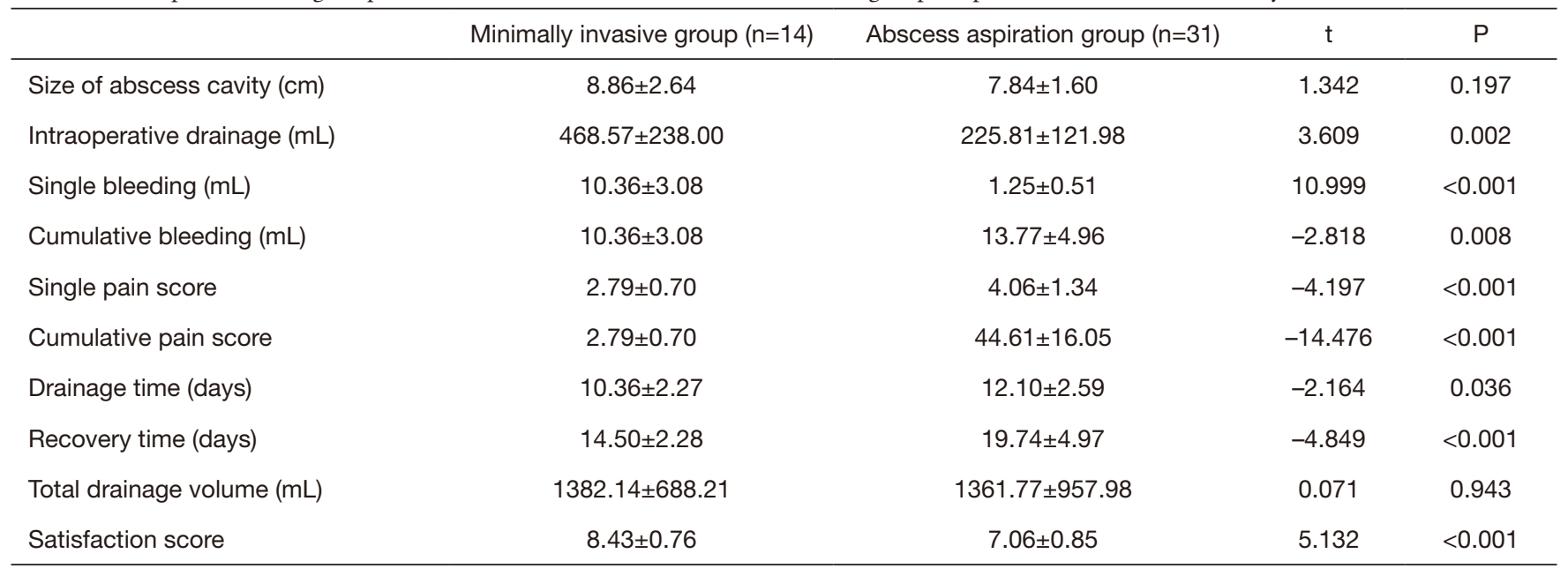

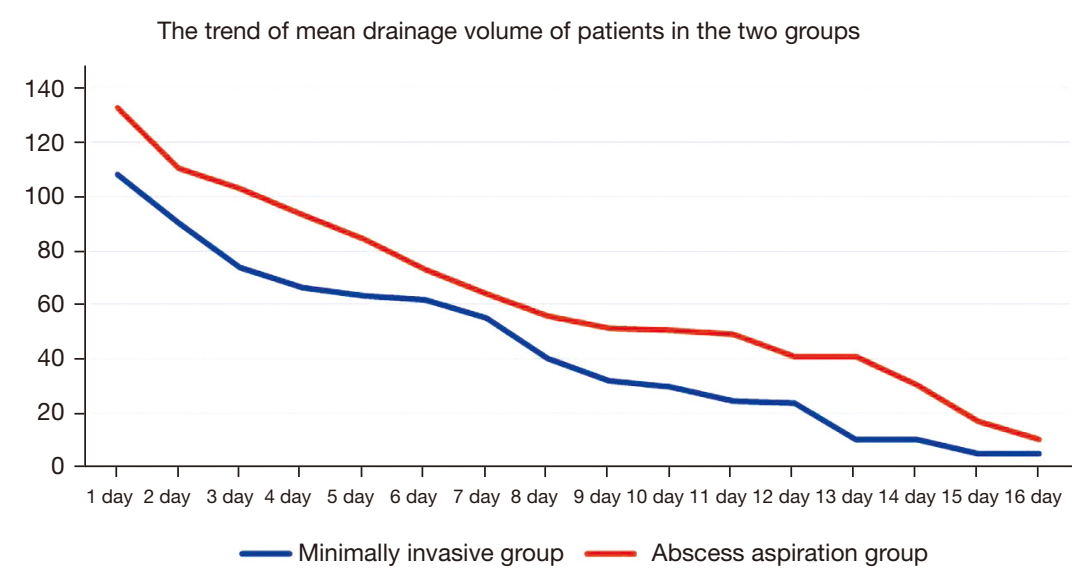

Figure 2 The mean drainage volume trends of patients in the 2 groups. In the minimally invasive group, the drainage time was $2-$ $16(7.17 \pm 3.35)$ days, and the minimum drainage volume was $5 \mathrm{~mL}$. In the abscess aspiration group, the drainage time was $1-$ $16(9.56 \pm 3.99)$ days, and the minimum drainage volume was $10 \mathrm{~mL}$. The total amount of drainage recorded in this study was the sum of the intraoperative drainage plus the postoperative drainage.

the patients with different abscess cavity diameters into groups, and the results revealed that minimally invasive rotary resection had significant advantages in the treatment of large abscesses (with a cavity diameter $>5 \mathrm{~cm}$ ) while puncture abscess aspiration may be more appropriate in the treatment of small abscesses (with a cavity diameter $\leq 5 \mathrm{~cm}$ ). Our study provides new evidence that should assist surgeons to make better clinical decisions when treating patients with lactational breast abscess.

According to previous reports, about $70.6 \%$ of women develop a breast abscess after first delivery, and the time interval for abscess onset is about 42 days (14). Common symptoms for the occurrence of breast abscess include sore nipples or breast engorgement (15). Many risk factors have been shown to be related to the development of a breast abscess, including race, smoking, obesity, and bacteria and other micro-organism infections (16). As breast abscesses cause serious complications in lactating women, it is essential that they are appropriately treated. Abscess drainage has been confirmed to be the most effective strategy for the removal of an abscess (7), but the methods used to achieve this vary. In the early days, incisive drainage was widely used, and satisfactory clinical outcomes (i.e., a high recovery rate and a low relapse rate) 
were achieved (17). However, this method has gradually become obsolete, as it has a number of disadvantages, including a long healing time, pain associated with dressing the wound, poor cosmetic effects, and the disruption of breastfeeding (18). Ultrasound-guided abscess puncture and drainage can replace surgical incision and drainage, and can basically cure most breast abscesses $(19,20)$. However, it also has some shortcomings, such as a poor aspiration effect and a high relapse rate. Thus, finding more appropriate methods represents a new challenge in the optimal treatment of lactational breast abscess.

In recent years, the vacuum-assisted breast biopsy (VABB) system has been explored in the treatment of mastitis and found to have good clinical effects. The VABB system is a clinical biopsy system that was developed by Fred Burbank and Mark Retchard in 1995 (21). It is mainly composed of a rotary cutter and vacuum pump. Under the guidance of ultrasound, the rotary cutter is percutaneously inserted into the breast lesions to dissect the lesions, and the lesions are then sucked out using negative pressure. Under this procedure, the histological specimens of the breast lesions are obtained. Breast lesions can be cut repeatedly, and the $\mathrm{VABB}$ can cut multiple specimens in 1 puncture (22-24). As it has a rotary cutter, the VABB system can puncture almost any part of a breast lesion, and thus has certain advantages over traditional surgery (25).

In this study, all the patients were outpatients and did not require hospitalization. The antibiotics used during the treatment were mainly first-generation cephalosporin (cefradine) and second-generation cephalosporin (cefuroxime). If a patient was allergic to cephalosporin, lincosamide antibiotics (clindamycin) were used. During antibiotic infusion, patients can continue to breastfeed, but the baby must be strictly observed for gastrointestinal symptoms (26).

In this study, the patients were divided into a minimally invasive group and an abscess aspiration group. After surgery, the drainage time and complete recovery time of patients in the minimally invasive group were shorter than those in the abscess aspiration group, but the total amount of drainage was similar between the 2 groups. Patients in the minimally invasive group had higher satisfaction scores, but their postoperative scars were also slightly longer. The 2 treatment methods did not affect postoperative recurrence or the continuance of breastfeeding. The results of an intraoperative questionnaire survey revealed that $93.75 \%(75 / 80)$ of the patients indicated they were willing to continue breastfeeding, and these patients were aware of the importance of breastfeeding. In this study, we did not recommend that patients stop breastfeeding after surgery.

The above results did not consider the size of the abscess cavity and the difficulty of abscess aspiration. In fact, cavity size is an important factor affecting the selection of the treatment method and patient outcomes (27), which we have also confirmed in clinical practice. Li et al. (28) compared the efficacy of minimally invasive rotary resection plus catheter drainage and traditional surgical abscess incision, and proposed that the indication for the minimally invasive method was an abscess cavity diameter $\geq 3 \mathrm{~cm}$. Eryilmaz and colleagues found that the efficacy of abscess aspiration was not obvious when the diameter of the abscess cavity was $>5 \mathrm{~cm}(13)$.

Based on the findings of the present study, a number of conclusions can be drawn. First, we will set out our findings in relation to the pain score and amount of bleeding. Due to the narrow operation space of a small abscess cavity, we found that it was easy to touch the wall of the abscess cavity using the minimally invasive technique, and the pain of patients with a small abscess cavity was more obvious than that of those with a large abscess cavity. A 20 or $50 \mathrm{~mL}$ syringe was used to suck the abscess in the abscess aspiration group. As the puncture can basically complete the absorption of the abscess, the single pain score in the abscess aspiration group was lower than that in the minimally invasive group. Due to the large operation space of a large abscess cavity, the rotary cutter was easy to absorb and did not touch the cavity wall using the minimally invasive technique; thus, patients' pain during the operation was not obvious. Since the minimally invasive group only needed 1 puncture operation, and the silicone drainage tube was retained for drainage in the later period, the drainage fluid caused no obvious bleeding and the pain was not significant. Puncture and aspiration required repeated punctures. The single pain score and bleeding volume were low; however, the cumulative pain score and bleeding volume of the abscess aspiration group were higher than those of the minimally invasive group after repeated punctures. Thus, in terms of pain and bleeding, the use of the minimally invasive treatment in patients with a large abscess cavity had obvious advantages. Thus, a large abscess cavity is suitable for minimally invasive rotary resection plus catheter drainage.

Second, we will set out our findings in relation to the amount of drainage. For the large abscess cavity, the minimally invasive technique sucked more abscess during 
the operation, and the abscess cavity was in a state of continuous negative pressure suction after surgery, and the abscess cavity attached well, so the daily postoperative drainage volume of the patient was lower than that of the abscess aspiration group. In the abscess aspiration group, each abscess aspiration was not as thorough as each minimally invasive abscess aspiration, as there were always residues. During the interval between each abscess aspiration, the bacteria have a chance to proliferate and the amount of abscess can increase. This may be one reason why the decrease of abscess volume in the abscess aspiration group was relatively slow, and the amount of daily postoperative drainage was relatively large. Thus, the drainage in the minimally invasive group with the large abscess cavity was more thorough, which reduced the chance of residue and recurrence, and has obvious advantages. It was suitable for minimally invasive rotary resection plus catheter drainage.

In addition, for deep abscesses and multiple abscesses, the effect of abscess aspiration was poor. Further, when a patient's breast was large, the syringe needle could not even penetrate into the abscess cavity, and the effect of abscess aspiration was extremely poor.

\section{Limitations}

This study had some limitations. First, it was a retrospective study and was conducted at a single center. Prospective cohort studies at multiple centers need to be conducted to confirm the conclusions drawn in this study. Second, the sample size of our cohort was relatively small, especially in the subgroup analysis of patients with different abscess cavity diameters. We will expand the sample size by collecting the data of more patients with lactational breast abscess in our clinical practices. Third, the long-term outcomes of patients were not investigated; rather, we only observed the intraoperative and postoperative indicators. We will explore the long-term outcomes in our future studies.

\section{Conclusions}

In conclusion, we believe that when the abscess cavity is relatively small (has a diameter $\leq 5 \mathrm{~cm}$ ) and the abscess is superficial, puncture and abscess aspiration may be more advantageous than the minimally invasive rotary resection plus catheter drainage. When an abscess cavity is relatively large (has a diameter $>5 \mathrm{~cm}$ ), and there are multiple abscesses or a deep large abscess, the advantages of minimally invasive rotary resection plus catheter drainage are more obvious. The treatment effect is definite and worthy of clinical promotion.

\section{Acknowledgments}

Funding: None.

\section{Footnote}

Reporting Checklist: The authors have completed the STROBE reporting checklist. Available at https://dx.doi. org/10.21037/gs-21-729

Data Sharing Statement: Available at https://dx.doi. org/10.21037/gs-21-729

Conflicts of Interest: All authors have completed the ICMJE uniform disclosure form (available at https://dx.doi. org/10.21037/gs-21-729). The authors have no conflicts of interest to declare.

Ethical Statement: The authors are accountable for all aspects of the work in ensuring that questions related to the accuracy or integrity of any part of the work are appropriately investigated and resolved. All procedures performed in this study involving human participants were in accordance with the Declaration of Helsinki (as revised in 2013). The study was approved by ethics committee of Tianjin Central Hospital of Gynecology Obstetrics (No. $2021 \mathrm{KY} 058$ ) and informed consents were exempted by the ethics committee due to the retrospective nature of this study.

Open Access Statement: This is an Open Access article distributed in accordance with the Creative Commons Attribution-NonCommercial-NoDerivs 4.0 International License (CC BY-NC-ND 4.0), which permits the noncommercial replication and distribution of the article with the strict proviso that no changes or edits are made and the original work is properly cited (including links to both the formal publication through the relevant DOI and the license). See: https://creativecommons.org/licenses/by-ncnd/4.0/.

\section{References}

1. Bosma MS, Morden KL, Klein KA, et al. Breast imaging 
after dark: patient outcomes following evaluation for breast abscess in the emergency department after hours. Emerg Radiol 2016;23:29-33.

2. Kong C, Zhang C, Wu Y, et al. The expression and meaning of CD68, CD163, CD57, and IgG4 in granulomatous lobular mastitis. Gland Surg 2020;9:936-49.

3. Dener C, Inan A. Breast abscesses in lactating women. World J Surg 2003;27:130-3.

4. Sun K, Chen M, Yin Y, et al. Why Chinese mothers stop breastfeeding: Mothers' self-reported reasons for stopping during the first six months. J Child Health Care 2017;21:353-63.

5. Amir LH. Academy of Breastfeeding Medicine Protocol Committee. ABM clinical protocol \#4: Mastitis, revised March 2014. Breastfeed Med 2014;9:239-43.

6. Chen C, Luo LB, Gao D, et al. Surgical drainage of lactational breast abscess with ultrasound-guided Encor vacuum-assisted breast biopsy system. Breast J 2019;25:889-97.

7. Kataria K, Srivastava A, Dhar A. Management of lactational mastitis and breast abscesses: review of current knowledge and practice. Indian J Surg 2013;75:430-5.

8. Wang X, Ning P, Ma XJ. Guidelines for diagnosis and treatment of breast-feeding mastitis in China. Chinese Journal of Breast Disease (Electronic Version) 2020;14:10-14.

9. Kang YD, Kim YM. Comparison of needle aspiration and vacuum-assisted biopsy in the ultrasound-guided drainage of lactational breast abscesses. Ultrasonography 2016;35:148-52

10. Liu YZ, Liu JL, Chen JA, et al. Clinical effect of ultrasound guided minimally invasive surgical treatment of non-lactating mastitis. Chinese Journal of Breast Disease (Electronic Version) 2018;12:360-4.

11. Ning P, Llu ZY, Chen J. Study on clinical classification and individualized treatment strategy of breast-feeding mastitis. Chinese Journal of Breast Disease (Electronic Version) 2013;7:7-11.

12. Wang L, Sun XJ, Yang WD, et al. A comparative study of the clinical effects of vacuum drainage and traditional open surgery for small incisions. Systems Medicine 2017;2:68-70.

13. Eryilmaz R, Sahin M, Hakan Tekelioglu M, et al. Management of lactational breast abscesses. Breast 2005;14:375-9.

14. Boccaccio C, Verdaguer Babic V, Botto L, et al. Methicillin-resistant Staphylococcus aureus (MRSA) isolation in breast abscesses in a Public Maternity. Medicina (B Aires) 2014;74:210-5.

15. Egbe TO, Njamen TN, Essome H, et al. The estimated incidence of lactational breast abscess and description of its management by percutaneous aspiration at the Douala General Hospital, Cameroon. Int Breastfeed J 2020;15:26.

16. Bharat A, Gao F, Aft RL, et al. Predictors of primary breast abscesses and recurrence. World J Surg 2009;33:2582-6.

17. Fahrni M, Schwarz EI, Stadlmann S, et al. Breast Abscesses: Diagnosis, Treatment and Outcome. Breast Care (Basel) 2012;7:32-8.

18. Hussain N, Khan I, Ahmed T, et al, Comparison of the restoration of breastfeeding after percutaneous aspiration vs incision and drainage for management of breast abscess. J Liaquat Univ Med Health Sci 2018;17:47-51.

19. Naeem M, Rahimnajjad MK, Rahimnajjad NA, et al. Comparison of incision and drainage against needle aspiration for the treatment of breast abscess. Am Surg 2012;78:1224-7.

20. Colin C, Delov AG, Peyron-Faure N, et al. Breast abscesses in lactating women: evidences for ultrasoundguided percutaneous drainage to avoid surgery. Emerg Radiol 2019;26:507-14.

21. Innocenti A, Melita D. The Vacuum-Assisted Breast Biopsy System is an Effective Strategy for the Treatment of Gynecomastia. Aesthetic Plast Surg 2021. [Epub ahead of print]. doi: 10.1007/s00266-020-02053-x.

22. Evans A, Whelehan P, Warwick V, et al. Percutaneous sentinel node removal using a vacuum-assisted needle biopsy in women with breast cancer: a feasibility and acceptability study. Clin Radiol 2019;74:216-9.

23. Heil J, Richter H, Golatta M, et al. Vacuum-Assisted Biopsy to Diagnose a Pathological Complete Response in Breast Cancer Patients After Neoadjuvant Systemic Therapy. Ann Surg 2018;268:e60-1.

24. Park HL, Hong J. Vacuum-assisted breast biopsy for breast cancer. Gland Surg 2014;3:120-7.

25. Liu YK, Huang XY, Weng YY, et al. Application of minimally invasive rotary resection in the treatment of deep breast abscess. Chin J Gen Surg 2015;24:1601-4.

26. Gu YS, Ye JM, Expert advice on diagnosis and treatment of breast-feeding mastitis. Chin Clin Dr 2019;47:1276-81.

27. Kousar N, Durrani TA, Ghafoor T, et al. Large lactational breast abscess: primary closure with drain versus conventional incision and drainage. JSZMC 2018;9:1439-42. 
28. Li ZF, Chen JX, Sun JJ. Surgical method and experience of Mammotome minimally invasive surgical treatment of breast abscess during lactation. Journal of Nantong

Cite this article as: Fu ZL, Zhang L, Feng R, Wan FX. Clinical research on minimally invasive rotary resection in the treatment of lactational breast abscess. Gland Surg 2021;10(12):3294-3304. doi: 10.21037/gs-21-729
University (Medical Edition) 2018;38:230-2.

(English Language Editor: L. Huleatt) 